\title{
Therapieoptionen zur Rekonstruktionen der dorsalen Rumpfwand
}

\author{
Therapeutic Options for Reconstruction of the Dorsal Trunk Wall
}

\author{
Autoren \\ B. Behr, T. Hirsch, O. Goertz, A. Ring, M. Lehnhardt, A. Daigeler \\ Institut \\ Klinik für Plastische Chirurgie, Universitätsklinikum Bergmannsheil Bochum, Bochum
}

\section{Schlüsselwörter \\ ○ Rücken \\ - freie Lappenplastiken \\ - gestielte Lappenplastiken \\ - Perforans- Lappenplastiken \\ - Rekonstruktion}

Key words

posterior trunk

- free flaps

- pedicled flaps

- reconstruction perforator flaps

\section{Zusammenfassung}

Defekte der dorsalen Rumpfwand stellen herausfordernde rekonstruktive Probleme dar, welche aufgrund der speziellen Anatomie die Beherrschung verschiedener Techniken erfordern. In Abhängigkeit von der Lokalisation können Defektdeckungen an der dorsalen Rumpfwand mit einfachen Verschiebeschwenklappenplastiken, Propellerlappenplastiken, gestielten Lappenplastiken und freien Lappenplastiken durchgeführt werden. Die Mehrzahl der Defekte kann mit gestielten Verfahren verschlossen werden. Besteht die Indikation zur Defektdeckung mittels freier Lappenplastik, kann ein Gefäßinterponat den Anschluss erleichtern.

Mit Ausnahme der M. latissimus dorsi-Umkehrlappenplastik konnte im eigenen Patientengut mit sämtlichen Verfahren ein suffizienter Defektverschluss erreicht werden. Basierend auf der anatomischen Lokalisation stellen wir anhand unserer Erfahrungen einen Therapiealgorithmus zur Defektdeckung am der dorsalen Rumpfwand auf.

eingereicht 20.2.2014 akzeptiert 24.2.2014

Bibliografie

DOI http://dx.doi.org/ 10.1055/s-0034-1370994 Handchir Mikrochir Plast Chir 2014; 46: 90-96 (c) Georg Thieme Verlag KG Stuttgart · New York ISSN 0722-1819

Korrespondenzadresse PD Dr. med. Björn Behr Klinik für Plastische Chirurgie Universitätsklinikum Bergmannsheil Bochum Bürkle-de-la-Camp-Platz 1 44789 Bochum bjorn.behr@rub.de

\section{Einleitung}

Weichteildefekte der dorsalen Rumpfwand stellen eine besondere Herausforderung für den rekonstruktiv tätigen Chirurgen dar. Traumata, Tumoren, Strahlenschäden, kongenitale Fehlbildungen wie Spina bifida oder postoperative Wundheilungsstörungen nach Wirbelsäuleneingriffen führen zu diesen Defekten. Bei der Behandlung von Defekten der dorsalen Rumpfwand müssen zunächst die üblichen chirurgischen Grundprinzipien wie das radikale Debridement und eventuell eine Konditionierung des Wundgrundes mit Vakuumtherapie eingehalten werden. Ebenso sollten osteosynthetische Verfahren an der Wirbelsäule erfolgt sein. Processus spinosi dorsales können und sollten, so sie im Defekt lie-

\section{Abstract}

Posterior trunk defects represent a major reconstructive problem, which require the entire armamentarium of the reconstructive surgeon. Given the special anatomy of the back, flap selection includes transposition flaps, perforator flaps, muscle flaps, turn-over flaps and free flaps, eventually with interposition of vein grafts. The majority of the defects can be closed with pedicled muscle or musculocutaneous flaps. In our patient collective, sufficient closure could be obtained with all procedures, except the latissimus dorsi turn-over flap. Based on our clinical experience, we propose an algorithm for closure of posterior trunk defects related to the anatomical region.

gen, problemlos entfernt werden, damit sie nicht die darüber liegende Lappenplastik durch Druckulzeration gefährden. Therapiekonzepte wie Sekundärheilung oder Spalthauttransplantationen führen in dieser Region nur selten zu dauerhaftem Erfolg [1]. Neben der oft geringen Verschieblichkeit des Gewebes liegen die Herausforderungen ebenso in der speziellen Anatomie mit einer spärlichen Anzahl von potentiellen mikrochirurgischen Anschlussgefäßen [2].

Allerdings sind an der dorsalen Rumpfwand auch mehrere Muskeln lokalisiert, welche an ihrem Gefäßstiel transferiert werden können. Neben den etablierten Techniken wie gestielten M. latissimus dorsi-, M. trapezius-, und ParascapularLappenplastiken können in Ausnahmefällen auch freie Lappenplastiken mit Gefäßinterponaten 
Anwendung finden. Neuere Entwicklungen liegen in der Verwendung von fasziokutanen Perforans-Lappenplastiken der dorsalen Rumpfwand. Gerade in dieser Region sind eine Vielzahl von Perforatoren vorhanden. Erwähnenswert ist, dass im Kontext der Defektdeckung der dorsalen Rumpfwand zum ersten Mal das Konzept der „perforator-basierten“ fasziokutanen Defektdeckung beschrieben wurde [3]. In dem folgenden Übersichtsartikel werden verschiedene Techniken zur Defektdeckung am der dorsalen Rumpfwand beschrieben und anhand eigener Fallbeispiele illustriert.

\section{Verschiebeschwenk- und Propeller-Lappenplastiken} Die dorsale Rumpfwand bietet mit ihrer großen Fläche und einer Vielzahl von Perforatoren diverse Möglichkeiten der plastischchirurgischen Defektdeckung durch Verschiebeschwenk- und Propellerlappenplastiken. Hierbei ist wie auch an anderen Kör- perregionen die Einhaltung von bewährten Prinzipien, wie einem ausreichenden Breiten-Längenverhältnis bei Verschiebeschwenklappen ( $\bullet$ Abb. $\mathbf{1}$ ), sowie der richtige Größe des Angiosoms bei Perforans-Lappenplastiken ( $\bullet$ Abb. 2) notwendig. Herauszuheben ist, dass die Möglichkeit dieser Lappenplastiken stark von der Beschaffenheit des umliegenden Gewebes abhängt. Sollte hier eine Bestrahlung stattgefunden haben ist die Verwendung dieser Lappenplastiken eher kritisch zu werten. Gerade bei Defekten der dorsalen Rumpfwand können diese Propeller-Lappenplastiken jedoch aufgrund der vielen Perforatoren von hohem Nutzen sein [4]. Hervorzuheben ist, dass mit diesen Operationsverfahren die Muskeln geschont werden und damit noch für weitere Rekonstruktionen zur Verfügung stehen. Für die operative Planung ist hier ein einfaches Handdopplergerät sehr hilfreich. Darüberhinaus ist auch die Kombination beider Verfahren möglich. So kann ein Perforator ohne gesonderte
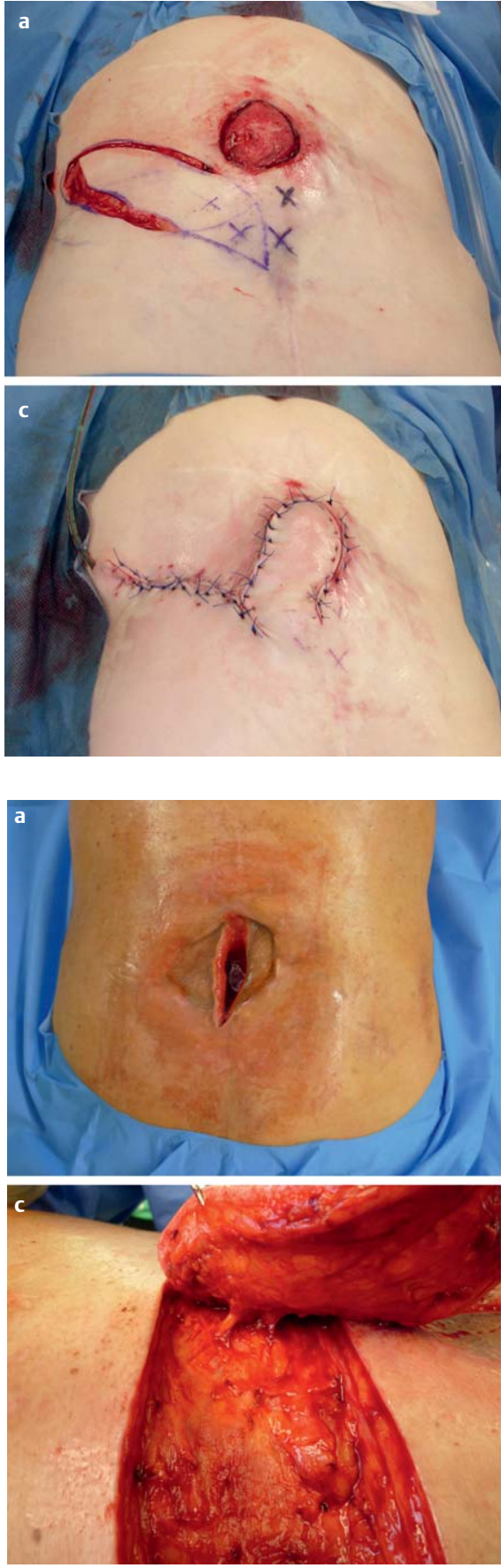
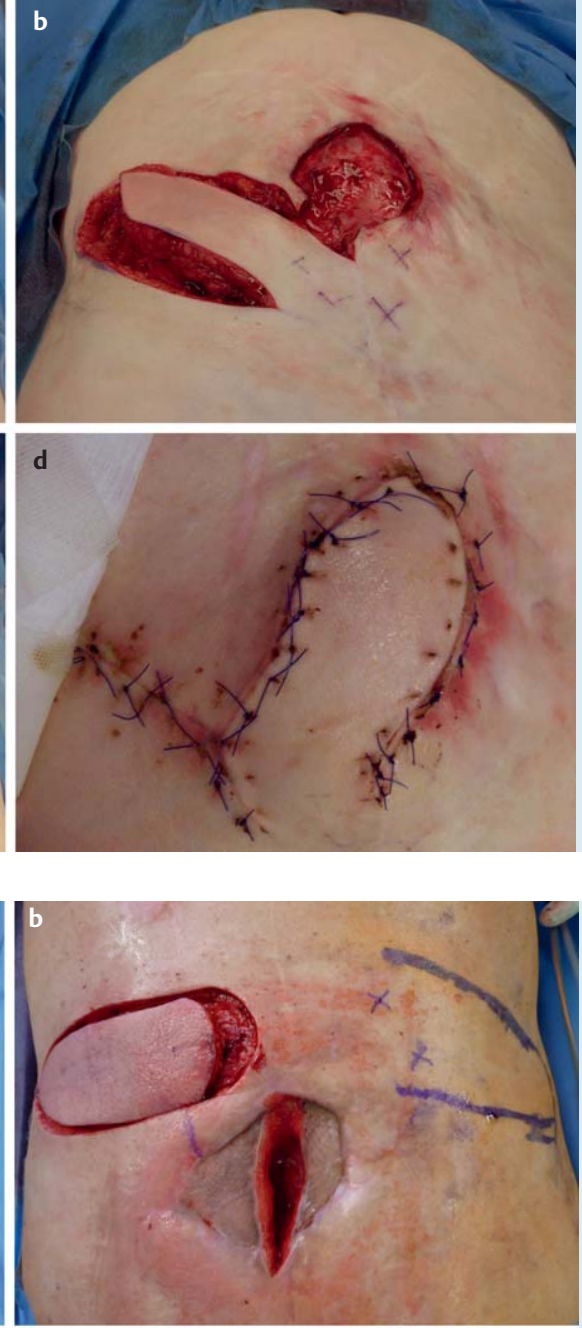

Abb. 2 Fasziokutane Propeller-Lappenplastik nach erfolglosem Versuch der lumbalen Spalthautdeckung (a präoperativer Status; b präparierte und gut vaskularisierte Propeller-Lappenplastik vor dem Schwenkmanöver; c Darstellung des Perforators der Propeller-Lappenplastik; d eingenähte Propeller-Lappenplastik).

Abb. 1 Verschiebeschwenklappenplastik (VSL) im Lumbalbereich mit integriertem Perforator (a VSL in situ mit angezeichneten und zuvor identifizierten Perforatoren; b VSL vor der Rotation; c VSL nach der Einnaht; $\mathbf{d}$ postoperatives Bild).

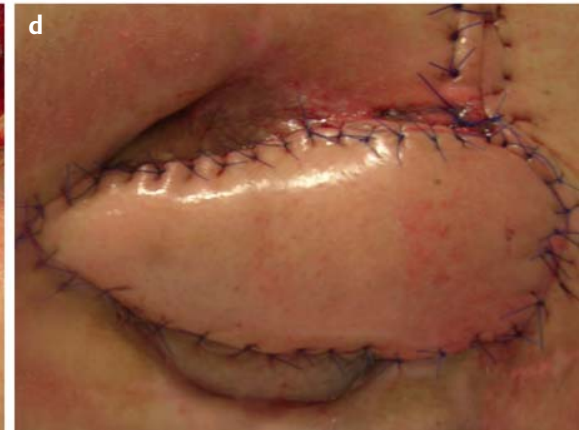


Präparation in das Zentrum eines Verschiebeschwenklappens gelegt werden um dann größere und sicherere Verschiebeschwenklappen zu kreieren. Eine übliche Option der fasziokutanen Defektdeckung stellen die Scapular- oder Parascapular-Lappenplastiken dar. Diese werden an dem Ramus transversalis oder descendens der A. circumflexa scapulae gestielt und können Defekte im oberen und mittleren Rückenbereich verschließen. Ähnlich wie bei der Präparation als freie Lappenplastik kann der Stiel bis in die mediale Achsellücke verfolgt werden. Die Dimensionen der Parascapular-Lappenplastiken können mehr als $10 \times 30 \mathrm{~cm}$ betragen; sie ist damit die größere der beiden Lappenplastiken. Herauszuheben sind ferner gluteale Perforatorlappenplastiken zum Defektverschluss im Sakralbereich [5]. Hierbei kann z.B. beim Superior Gluteal Artery Perforator Flap (S-GAP) die A. glutealis superior ohne großflächige Schädigung des M. gluteus maximus präpariert werden ( $\bullet$ Abb. 3 ). Ferner können in dieser Region Lumbal- und Intercostalarterien basierte Perforans-Lappenplastiken ihre Anwendung finden [6].

\section{M. latissimus dorsi-Lappenplastik}

Der M. latissimus dorsi bietet mit seiner Größe von etwa $20 \times 40 \mathrm{~cm}$ die Möglichkeit sehr große Defekte zu decken und ist in seinem Anwendungsspektrum sehr vielseitig. Er kann muskulär oder muskulokutan zur Rekonstruktion von Defekten verwendet werden. Der Muskel entspringt dem hinteren Drittel der Crista iliaca, der 7.-12. Brustwirbel und der 9.-12. Rippe. Er setzt an der Crista tuberculi minoris des Humerus an. Gemäß der Einteilung nach Mathes und Nahai ist er ein Typ 5 Muskel und hat als dominantes Gefäß die A. thoracodorsalis, welche Teil des scapularen Gefäßbaums ist. Die nicht-dominanten Gefäße sind Perforatoren der Intercostal- und Lumbalarterien; bleiben sie gemeinsam erhalten ist eine Versorgung des Lappens über diese Gefäße möglich. Neben den verschiedenen Optionen die M. latissimus-Lappenplastik gestielt für ipsilaterale Defekte einzusetzen, ermöglichen letztgenannte Perforatoren auch die Defektdeckung von kontralateralen oder kaudal gelegenen Defekten beim Einsatz des Muskels als Umkipplappenplastik [7,8]. Ein häufiges Problem liegt hier jedoch in der Blutversorgung, da im Rahmen des Einsatz von Osteosynthesematerials oder der dor- salen Instrumentierung von Wirbelsäulen die lumbalen Perforatoren bereits zerstört sind. Aus diesem Grund sollte präoperativ das Vorliegen der Perforatoren geprüft werden. In unserem eigenen Patientengut sind die Erfahrungen mit der M. latissimusUmkehrlappenplastik durchwachsen und wir setzen ihn nicht mehr zur Defektdeckung ein. Als Alternative kann der M. latissimus unter dem Einsatz von Gefäßinterponaten für die Defektdeckung von weiter kaudal gelegenen Defekten verwendet werden ( $\bullet$ Abb. 4) [9]. Im Regelfall muss der großflächige Muskel dann noch mit Spalthaut bedeckt werden.

\section{Supraclaviculäre Lappenplastiken}

Supraclaviculäre Lappenplastiken, welche auch als Insellappenplastiken gehoben werden können, stellen eine weitere interessante fasziokutane Möglichkeit der Defektdeckung im Nackenbereich dar. Er wird aus der Schulterregion gehoben und von der A. cervicalis transversa versorgt. Das damit versorgte Angiosom beträgt mindestens $22 \mathrm{~cm}$ in der Länge und $10 \mathrm{~cm}$ in der Breite [10]. Auch in schwierigen, gefäßdepletierten chirurgischen Verhältnissen, in der Hals und Nackenregion kann mit dieser Technik ein zufriedenstellender Verschluss erreicht werden [11].

\section{M. trapezius-Lappenplastik}

Der Trapeziusmuskel gehört gemäß der Einteilung von Mathes und Nahai zur Gruppe 2 und eignet sich hervorragend zur Deckung von Defekten im oberen Rückenbereich. Er ist etwa $34 \times 18 \mathrm{~cm}$ groß und reicht vom Os occipitale bis zu den unteren Brustwirbeln (Th 12) und kann rein muskulär oder muskulokutan gehoben werden. Seine Blutversorgung erhält er aus dem Ramus ascendens und descendens der A. transversalis cervicalis und diversen intercostalen Perforatoren. Der Muskel kann von caudal nach cranial gehoben und in Defekte der oberen dorsalen Rumpfwand rotiert werden. Weitere Optionen bestehen in dessen Verwendung als Vorschub- oder Umkipplappenplastik [12-14]. Im gewählten Beispiel wurde die M-. trapezius-Lappenplastik muskulokutan zur Defektdeckung an der Schulter verwendet $(\bullet$ Abb. 5). Grundsätzlich eignet sich die Lappenplastik hervorragend zur Defektdeckung im oberen Rücken und Hals-/Schulterbereich $[2,15]$.
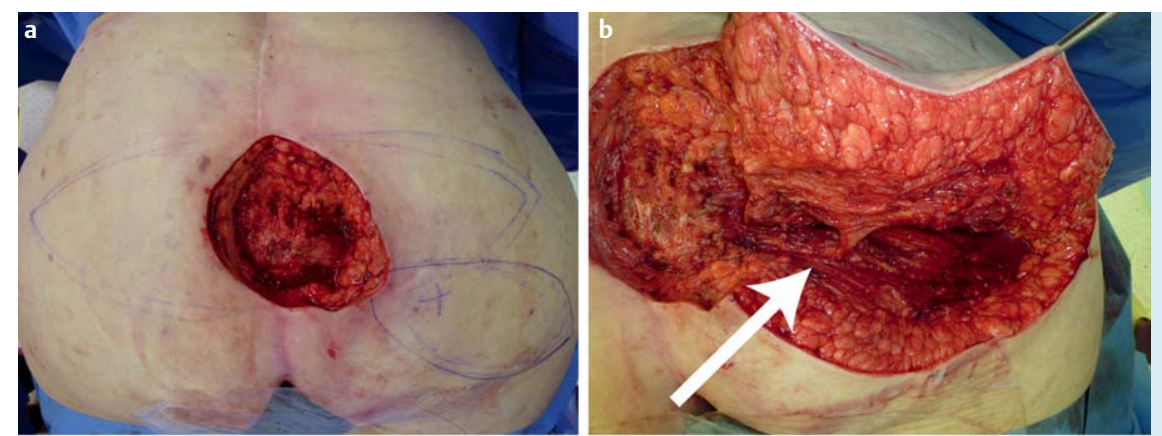

Abb. 3 Fasziokutane Superior Gluteal Artery Perforator Flap (S-GAP) Propellerlappenplastik zur Defektdeckung des Sakrums (a Status nach Debridement und Planung der Lappenplastik mit Einzeichnung des Perforators und Entscheidung gegen die Alternative von 2 VY-Lappenplastiken; b Präparation der S-GAP Lappenplastik und Skelettierung des Lappenstiels, der Pfeil zeigt auf den Lappenstiel; $\mathbf{c}$ rotierte Lappenplastik; $\mathbf{d}$ postoperativer Status).
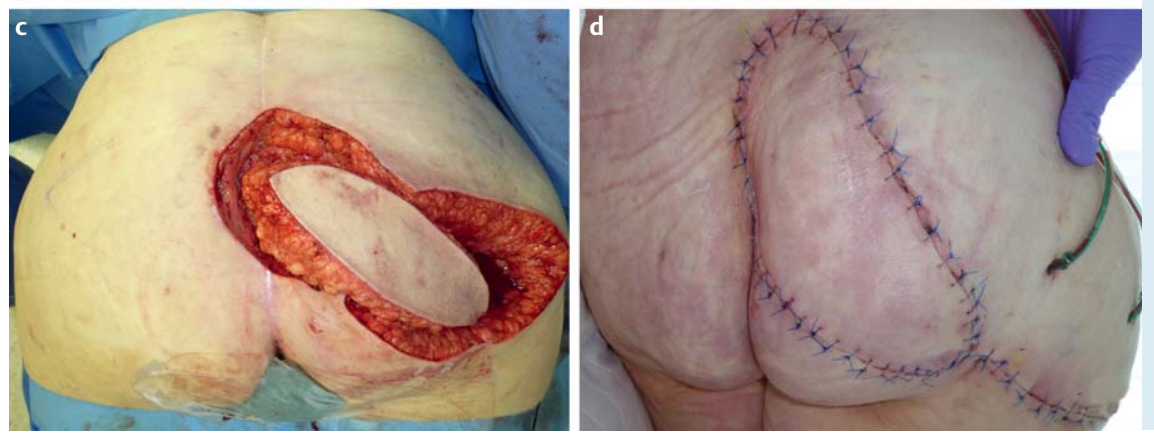

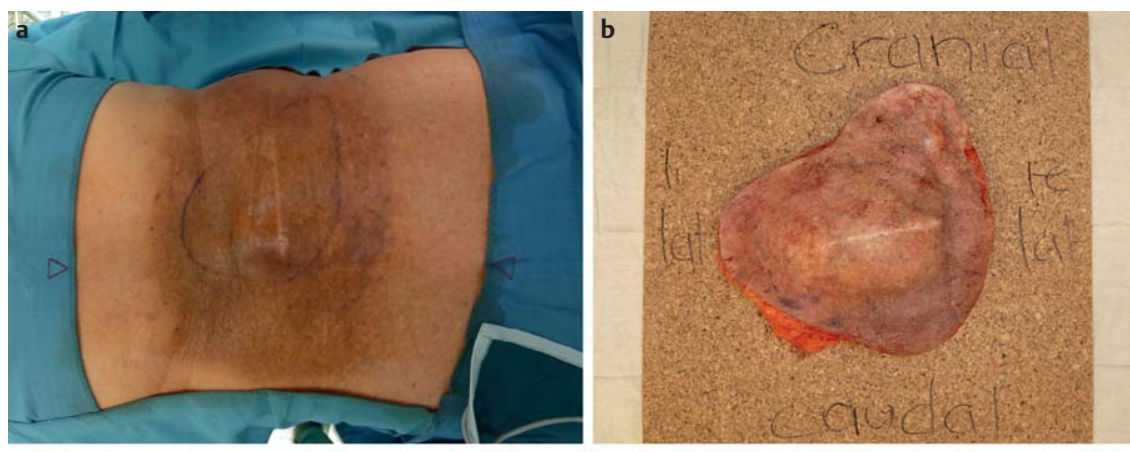

Abb. 4 NOS Sarkomresektion und Defektdeckung mit freier M. latissimus dorsi Lappenplastik unter der Verwendung von Gefäßinterponaten (a präoperativer Befund am Rücken mit eingezeichneten Tumor; b Resektat; c Operationssitus nach Resektion; $\mathbf{d}$ Operationssitus mit angeschlossenen Gefäßinterponaten an die A. thoracodorsalis und freier M. latissimus dorsi-Lappenplastik zur Defektdeckung im caudalen Rückenanteil; e Situs nach Hautverschluss über den Gefäßinterponaten; f: ausgeheilter Zustand 8 Wochen postoperativ).
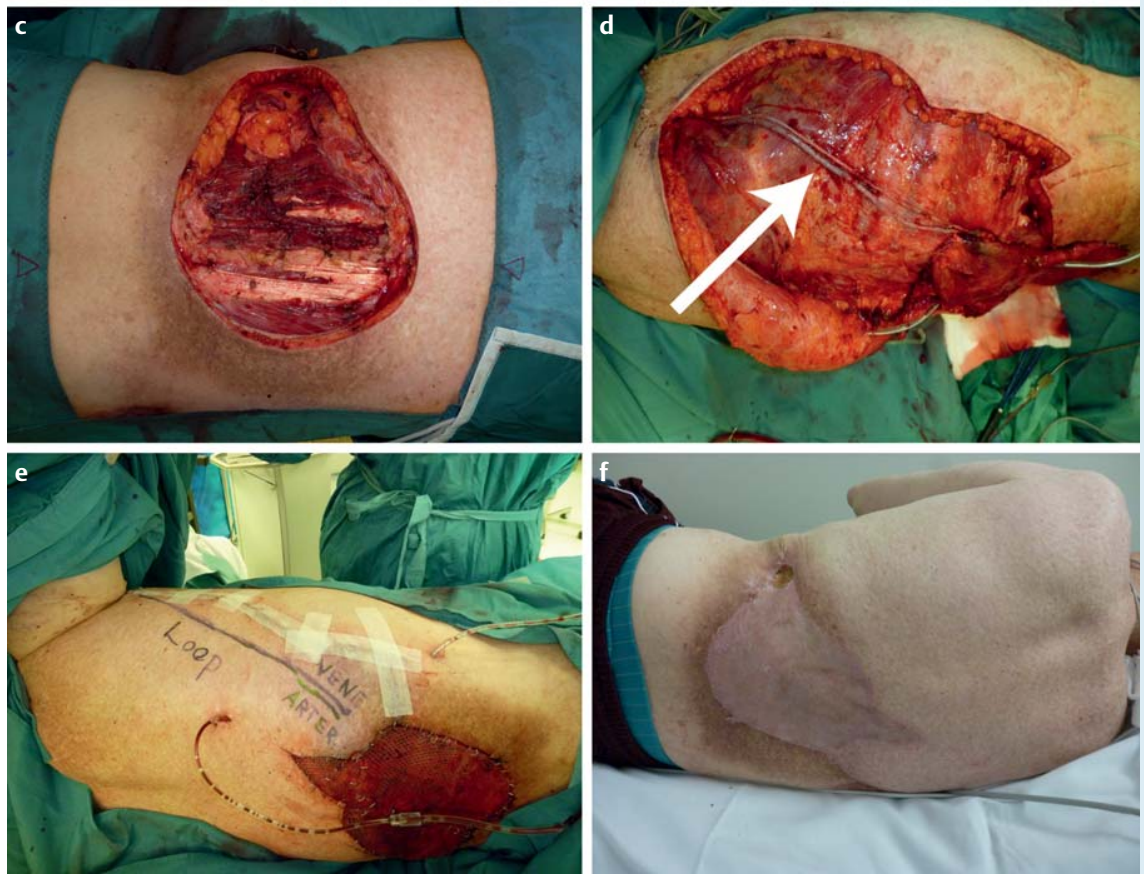

Paraspinale Muskellappenplastik

Als weitere Option für wirbelsäulennahe Defekte ist der paraspinale Muskellappen aufzuführen. Er ist im wesentlichen im Lumbalbereich, bis etwa zur Höhe Th10 einsetzbar und wird als Umkehrlappen verwendet $[2,16]$. Hierbei werden die Muskeln von den Processi transversi spinosi abpräperiert, nach medial verschoben und können so mit 2 gestielten Lappen spinale Wunden verschließen. Die medialen Perforatoren müssen getrennt werden, wohingegen die lateralen verschont bleiben.

\section{Durchzugs-VRAM}

Eine Option der Defektdeckung in der Sakralregion stellt der Durchzugs-VRAM (Vertical Rectus Abdominis Muscle) dar [17]. Hierbei kann der M. rectus abdominis Muskel mit Hautinsel an der A. epigastrica inferior gestielt und retroperitoneal getunnelt in den sakralen Defekt transponiert werden ( $\mathbf{A}$ Abb. 6).

\section{M. gluteus maximus-Lappenplastik}

Neben der Verwendung des fasziokutanen glutealen PerforansLappenplastik ist zur Defektdeckung im Sakralbereich auf die Rekonstruktion mit dem M. gluteus maximus möglich. Dieser Muskel reicht vom lateralen Sakrum bis zum Trochanter major und stellt einen Typ 3 Muskel mit 2 dominanten Gefäßen (A. glutealis superior und inferior) dar. Er kann insbesondere an seiner kranial gelegenen A. glutealis superior als Umkipplappenplastik in sakrale Defekte geschwenkt werden [18].
Mikrochirurgie und Möglichkeiten der Gefäßanschlüsse Sollte es nicht gelingen mit lokalen oder gestielten Lappenplastiken eine Defektdeckung an der dorsalen Rumpfwand zu erreichen, müssen freie Lappenplastiken erfolgen. Obwohl der Entnahme und der Wahl von Lappenplastiken hier keine Grenzen gesetzt sind, gestaltet sich der Gefäßanschluss an der dorsalen Rumpfwand als sehr schwierig. Als potentielles Anschlussgefäß kommt die A. glutealis superior am Gesäß in Frage [19]. Alternativ können Lappenplastiken ebenso an der vierten Lumbalarterie, lateral des M. sacrospinalis angeschlossen werden. Sollten diese Möglichkeiten nicht ausreichen, müssen Gefäßinterponate den Anschluss ermöglichen. Few und Kollegen berichten in diesem Zusammenhang über ihre mikrochirurgischen Erfahrungen im „feindlichen“ Rücken, definiert durch eine Defektgröße gröBer als $200 \mathrm{~cm}^{2}$, Strahlentherapie in der Anamnese, ausgeprägte Infektionen oder exponiertes Osteosynthesematerial [20]. Bei 4 Patienten erfolgte der Defektverschluss mit einer freien M. latissimus dorsi- oder VRAM-Lappenplastik mit Veneninterponaten. Hier wird typischerweise die Vena saphena magna verwendet [21]. Auch in unserem eigenen Patientengut hat sich diese Strategie als erfolgreich erwiesen ( $\bullet$ Abb. 4$)$.

\section{Wahl der Lappenplastik anhand der Lokalisation}

Anhand der anatomischen Lokalisation haben sich verschiedene Lappenplastiken bewährt ( $\bullet$ Abb. 7). Es ist selbstverständlich, dass die Wahl der Lappenplastik immer an die jeweilige Situa- 

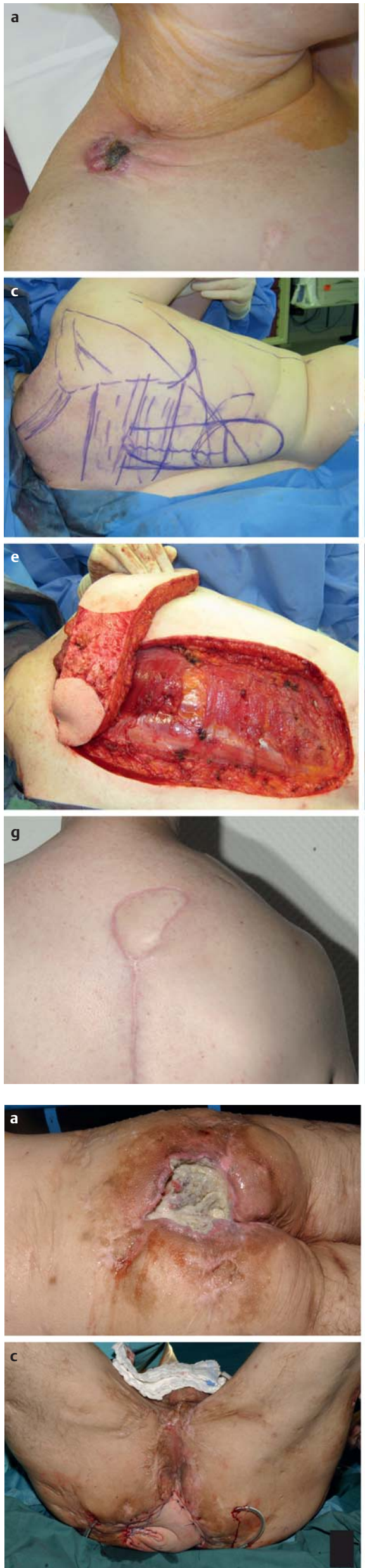
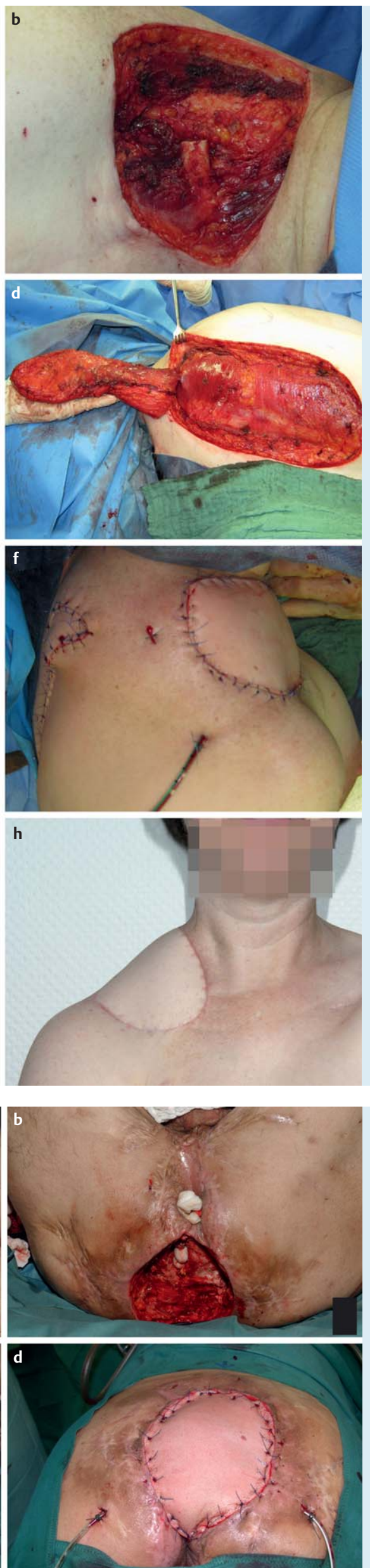

Abb. 5 Defektdeckung eines Weichteildefekts an der rechten Schulter mit einer muskulokutanen M. trapezius-Lappenplastik (a Metastase eines Mammakarzinoms an der rechten Schulter; b Zustand nach Resektion der Metastase mit freiliegender Clavicula; c Anzeichnung der M. trapezius-Lappenplastik inklusive der angrenzenden Strukturen; d Hebung der cranial gestielten M. trapezius-Lappenplastik; e geschwenkte M. trapezius-Lappenplastik mit 2 Hautinseln; f eingenähte $M$. trapezius-Lappenplastik; G Zustand 8 Wochen postoperativ von dorsal; h Zustand von ventral).

Abb. 6 Durchzugs-VRAM zur Defektdeckung im Sakralbereich (a präoperativer Befund bei Analkarzinom; b VRAM gestielt an der A. epigastrica inferior; c postoperativer Situs). 


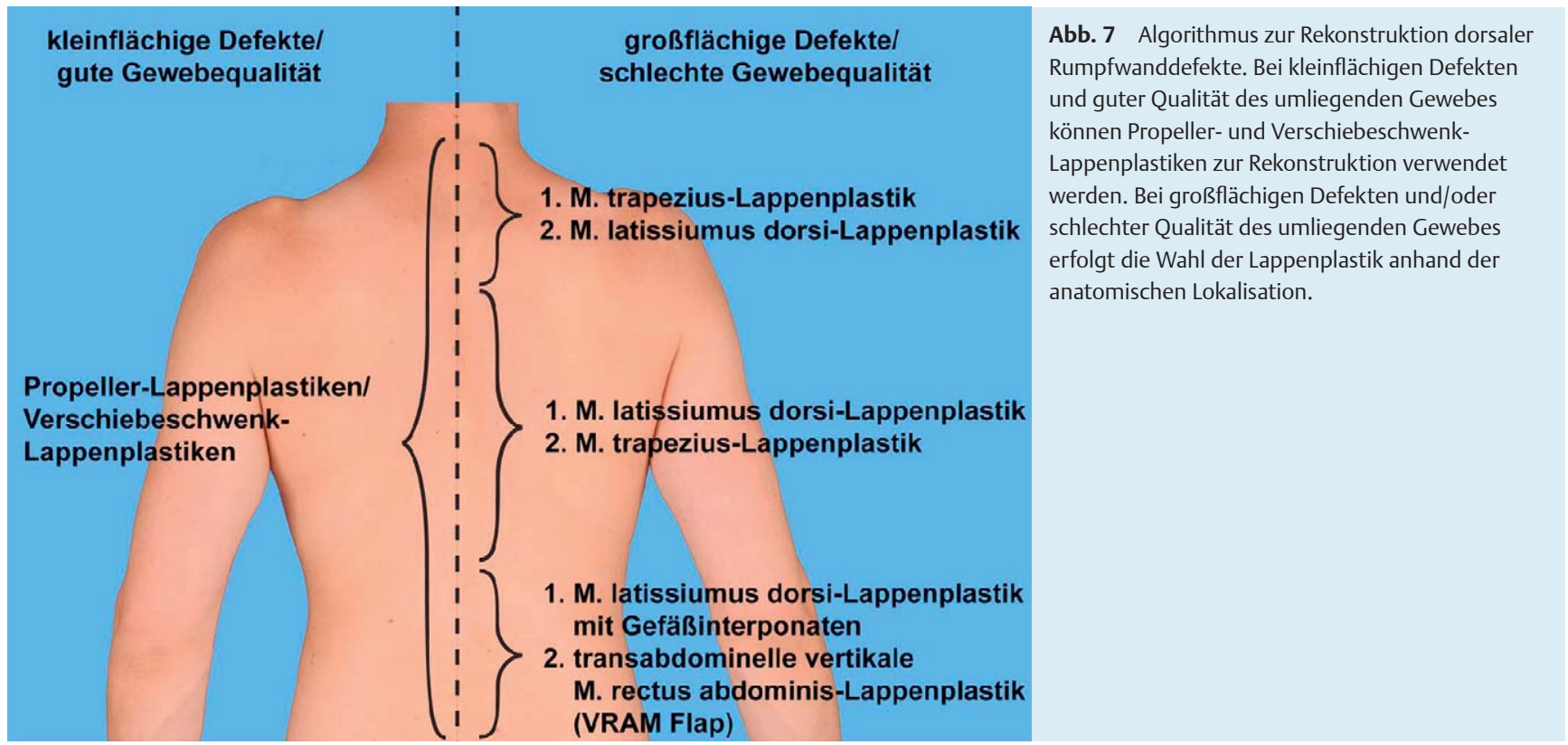

tion und das vorhandene Gewebe angepasst werden muss und dieser Algorithmus als Ausgangspunkt verstanden werden soll. In sämtlichen Regionen ist bei kleinflächigen Defekten und guter Qualität des umgebenden Gewebes der Verschluss mittels Propellerlappenplastiken und Verschiebeschwenklappenplastiken möglich. Darüberhinaus können je nach anatomischer Region die folgenden Lappenplastiken (auch in Kombination) Verwendung finden:

\section{Nacken- und Schulterregion}

Die Nacken- und Schulterregion stellt aufgrund der hohen Zugkräfte eine Herausforderung für die stabile Defektdeckung dar. Die Mehrzahl der Defekte lässt sich mit M. trapezius-Lappenplastiken decken. Hierbei lassen sich gute Ergebnisse erzielen, wenn die Hautinsel die Muskellappenplastik um nicht mehr als $1 \mathrm{~cm}$ überragt [22]. Sollte die M. trapezius-Lappenplastik aufgrund von Narben im Wundgebiet oder früheren Operationen, wie häufig in der Sarkomchirurgie üblich nicht mehr vorhanden sein, können zervikale Defekte allerdings auch mit M. latissimus dorsi-Lappenplastiken gedeckt werden.

\section{Obere Thoraxregion}

In der oberen Thoraxregion ist es sowohl möglich mit der M. latissimus dorsi aber auch mit der M. trapezius-Lappenplastik Defekte zu decken. Wenn möglich arbeiten wir aufgrund der hohen Sicherheit und umfangreichen Erfahrung gerne mit der M. latissimus dorsi-Lappenplastik. Beide Lappenplastiken können wahlweise als rein muskuläre mit Spalthaut oder muskulokutane Lappenplastiken verwendet werden. Selbstverständlich können beide Lappenplastiken auch in Kombination verwendet werden. Darüberhinaus haben auch die klassischen Verschiebeschwenk- oder Propellerlappenplastiken in der oberen Thoraxregion ihren Stellenwert.

\section{Untere Thoraxregion/Lumbalregion}

In der unteren Thoraxregion/Lumbalregion sind die Therapieoptionen oftmals schwieriger. Der M. latissimus reicht häufig in der Länge nicht aus, sodass bei der Verwendung dieses Muskels Verlängerungen nach kaudal unter der Verwendung von Gefäßinterponaten notwendig sind. Aus unserer Erfahrung raten wir von Umkipplappenplastiken ab. Darüberhinaus hat sich im Sakralbereich der Durchzugs-VRAM bewährt. Weitere Therapieoptionen liegen in der Verwendung der Paraspinalen Muskellappenplastik zum Verschluss von lumbalen Defekten, M. gluteus maximus-Lappenplastik bei sakralen Defekten, sowie von weiteren freien Lappenplastiken.

\section{Schlussfolgerung}

Defekte an der dorsalen Rumpfwand stellen aufgrund der speziellen Anatomie mit wenig Optionen für einen mikrochirurgischen Anschluss ein rekonstruktives Problem dar. Allerdings können die Mehrzahl der Defekte mit gestielten Lappenplastiken rekonstruiert werden. Werden die Grundprinzipien wie das radikale Debridement und die Entfernung von kontaminiertem Fremdmaterial beachtet, lassen sich mit den beschriebenen Methoden gute rekonstruktive Ergebnisse erzielen.

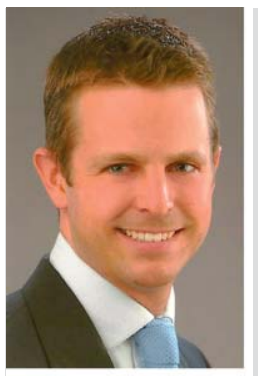

Björn Behr

1999-2005 Studium der Humanmedizin in Düsseldorf, Würzburg und Seattle. 2006 Promotion an der Universität Würzburg. 2006-2007 Assistenzarzt an der Klinik für Plastische Chirurgie, Universitätsklinikum Magdeburg (Prof. Dr. W. Schneider). 2007-2013 Assistenzarzt an der Klinik für Plastische Chirurgie, BG Unfallklinik Ludwigshafen (Prof. Dr. G.

Germann, Prof. Dr. M. Lehnhardt, Prof. Dr. U. Kneser). 2008-2010 Fellow im Department of Surgery, Division of Plastic Surgery an der Stanford Universität (Prof. Dr. M. T. Longaker). 2013 Habilitation an der Universität Heidelberg. Seit 2013 Oberarzt an der Klinik für Plastische Chirurgie, Universitätsklinikum Bergmannsheil Bochum (Prof. Dr. M. Lehnhardt). 


\section{Interessenkonflikt: Nein}

\section{Literatur}

1 Hallock GG. Reconstruction of posterior trunk defects. Seminars in plastic surgery 2011; 25: 78-85

2 Mathes DW, Thornton JF, Rohrich RJ. Management of posterior trunk defects. Plastic and reconstructive surgery 2006; 118: 73e-83e

3 Kroll SS, Rosenfield L. Perforator-based flaps for low posterior midline defects. Plastic and reconstructive surgery 1988; 81: 561-566

4 Hamdi M, Stillaert FB. Pedicled perforator flaps in the trunk. Clin Plast Surg 2010; 37: 655-665 vii

5 Koshima I, Moriguchi T, Soeda S et al. The gluteal perforator-based flap for repair of sacral pressure sores. Plastic and reconstructive surgery 1993; 91: 678-683

6 Roche NA, Van Landuyt K, Blondeel PN et al. The use of pedicled perforator flaps for reconstruction of lumbosacral defects. Annals of plastic surgery 2000; 45: 7-14

7 Stevenson TR, Rohrich RJ, Pollock RA et al. More experience with the "'reverse" latissimus dorsi musculocutaneous flap: precise location of blood supply. Plastic and reconstructive surgery 1984; 74: 237-243

8 Bostwick J $3^{\text {rd }}$, Scheflan M, Nahai F et al. The "reverse" latissimus dorsi muscle and musculocutaneous flap: anatomical and clinical considerations. Plastic and reconstructive surgery 1980; 65: 395-399

9 Hallock GG. An extended latissimus dorsi "non-free" flap. Br J Plast Surg 1987; 40: 516-517

10 Pallua N, Magnus Noah E. The tunneled supraclavicular island flap: an optimized technique for head and neck reconstruction. Plastic and reconstructive surgery 2000; 105: 842-851 discussion 852-844

11 Su T, Pirgousis P, Fernandes R. Versatility of supraclavicular artery island flap in head and neck reconstruction of vessel-depleted and difficult necks. J Oral Maxillofac Surg 2013; 71: 622-627

12 Mathes SJ, Stevenson TR. Reconstruction of posterior neck and skull with vertical trapezius musculocutaneous flap. Am J Surg 1988; 156: $248-251$
13 Lynch JR, Hansen JE, Chaffoo R et al. The lower trapezius musculocutaneous flap revisited: versatile coverage for complicated wounds to the posterior cervical and occipital regions based on the deep branch of the transverse cervical artery. Plastic and reconstructive surgery 2002; 109: 444-450

14 Disa JJ, Smith AW, Bilsky MH. Management of radiated reoperative wounds of the cervicothoracic spine: the role of the trapezius turnover flap. Annals of plastic surgery 2001; 47: 394-397

15 Ramasastry SS, Schlechter B, Cohen M. Reconstruction of posterior trunk defects. Clin Plast Surg 1995; 22: 167-185

16 Wilhelmi BJ, Snyder N, Colquhoun T et al. Bipedicle paraspinous muscle flaps for spinal wound closure: an anatomic and clinical study. Plastic and reconstructive surgery 2000; 106: 1305-1311

17 Daigeler A, Simidjiiska-Belyaeva M, Drucke D et al. The versatility of the pedicled vertical rectus abdominis myocutaneous flap in oncologic patients. Langenbeck's archives of surgery/Deutsche Gesellschaft für Chirurgie 2011; 396: 1271-1279

18 Koh PK, Tan BK, Hong SW et al. The gluteus maximus muscle flap for reconstruction of sacral chordoma defects. Annals of plastic surgery 2004; 53: 44-49

19 Park S, Koh KS. Superior gluteal vessel as recipient for free flap reconstruction of lumbosacral defect. Plastic and reconstructive surgery 1998; 101: 1842-1849

20 Few JW, Marcus JR, Lee MJ et al. Treatment of hostile midline back wounds: an extreme approach. Plastic and reconstructive surgery 2000; 105: 2448-2451

21 Earle AS, Feng LJ, Jordan RB. Long saphenous vein grafts as an aid to microsurgical reconstruction of the trunk. Journal of reconstructive microsurgery 1990; 6: 165-169

22 Chun JK, Lynch MJ, Poultsides GA. Distal trapezius musculocutaneous flap for upper thoracic back wounds associated with spinal instrumentation and radiation. Annals of plastic surgery 2003; 51: 17-22 